\title{
Neue Zielgruppe für Mentoring-Programme: Zugangserleichterung zum Hochschulstudium für ,nichttraditionelle Studierende' durch eine strukturierte Studieneingangsphase
}

\author{
Katrin Häuser
}

Eingegangen: 16. Juli 2015 / Angenommen: 11. November 2015 / Online publiziert: 30. November 2015 (C) Die Autor(en) 2015. Dieser Artikel ist auf Springerlink.com mit Open Access verfügbar.

Zusammenfassung Die Europäische Kommission strebt für 2020 die Steigerung der Hochschulabsolventen und -absolventinnen zwischen 30 bis 34 Jahren auf $40 \%$ an. Diese Anforderung stellt die Hochschulen vor neue Fragen an die Förderung dieser Zielgruppe. Aktuelle hochschuldidaktische Konzepte, wie Mentoring-Programme, können genutzt werden, um sich diesen Herausforderungen zu stellen. Der folgende Beitrag berichtet über Erkenntnisse in der Lernprozessbegleitung innerhalb des Mentoring-Programms „MEwiSA“ an der Universität Duisburg-Essen und greift Entwicklungsideen von Mentoring-Programmen auf. Der Artikel möchte vorläufige Empfehlungen geben, wie diese Erfahrungen aus dem Projekt genutzt werden können, um Hemmungen bei ,nicht-traditionellen Studierenden“, wie Lernenden im Erwachsenenalter, abzubauen und deren Studieneinstieg zu erleichtern.

Schlüsselwörter Mentoring $\cdot$ Abschlussbericht · Mentoring-Programme an Hochschulen $\cdot$ Studieneingangsphase $\cdot$ Nichttraditionelle Studienanfänger/innen

\begin{abstract}
One aim of the European Commission is to improve the number of graduates between 30 and 34 years to $40 \%$ for 2020 . This requirement provides the university with new material for the promotion of this target group. Current university didactic concepts, such as mentoring programs, can be used to address these challenges. The following article gives an overview of mentoring in higher education and reports on first experiences of participants in the mentoring program "MEwiSA" at the University of Duisburg-Essen. The intention of this article is to provide preliminary recommendations on how these experiences can be used to reduce inhibitions of "non-traditional students" and to facilitate their study entry.
\end{abstract}

K. Häuser, M.A. ( $\bowtie)$

Fachhochschule Südwestfalen,

Soest, Deutschland

E-Mail: haeuser.katrin@fh-swf.de 
Keywords mentoring $\cdot$ report $\cdot$ mentoring-programs at universities $\cdot$ the initial phase of the studies $\cdot$ non traditional students

\section{Einführung und Begriffsbestimmung}

Die Thematik „Mentoring“ ist ein aktuelles und mittlerweile etabliertes Instrument in der Erwachsenenbildung und Hochschuldidaktik. Ist von Mentoring die Rede, lässt sich zum allgemeinen Verständnis festhalten, dass es sich bei diesem Instrument um eine individuelle Beratungsform zwischen zwei Menschen handelt, in der sowohl persönliche wie berufliche Themen kommuniziert werden. Dieser Austausch mit einer erfahrenen Person hat eine Auswirkung auf die Persönlichkeit, die Fähigkeiten und den weiteren beruflichen Weg (vgl. Haasen 2001, S. 15 ff.). Die beteiligten Personen werden als Mentorin bzw. Mentor sowie Mentee bezeichnet.

Die erfahrenere Person fungiert als Mentorin bzw. Mentor, die synonym als Experte auf dem jeweiligen Gebiet betitelt werden kann. Eine Mentorin bzw. ein Mentor fungiert bewusst oder unbewusst als erlebbares Ziel. Diese Person hat das geschafft, wonach der/die Mentee strebt. Sei es eine Position oder eine Haltung, die erreicht werden möchte (vgl. Schell-Kiehl 2007, S. 18).

Die Entwicklung der bzw. des Mentee/s soll durch folgende Aufgaben der Mentorin bzw. des Mentors ermöglicht werden:

- Vermitteln wichtiger Informationen,

- Vermitteln von Erfahrungen,

- Vermitteln von Fähigkeiten,

- gezieltes Feedback der bzw. dem Mentee geben,

- dadurch Qualitäten und Potenziale offenlegen oder verbessern,

- emotionalen Beistand leisten

- (vgl. Schell-Kiehl 2005).

In manchen Fällen können ergänzend neben den typischen Aufgaben einer Mentorin oder eines Mentors auch noch gezielte Erwartungen an diese bzw. diesen formuliert werden. Dies ist abhängig von den Unternehmen oder Hochschulen.

Die oder der Mentee versteht sich hier als Person, der geholfen wird und die Unterstützung sowie Anerkennung durch eine erfahrenere Person erfährt. Wie bereits dargelegt, erlebt die oder der Mentee vor allem einen Vorteil durch die Entdeckung von Ressourcen und Potenzialen sowie der stabilen Ansprechpartnerin bzw. dem Ansprechpartner in Form der Mentorin bzw. des Mentors (vgl. Schlüter 2010, S. 102).

Die Hochschuldidaktik hat Mentoring-Programme entwickelt, um systematisch Studienanfängerinnen und -anfänger mit erfahreneren Studierenden zusammenzubringen. Hier kann das Mentoring als eine eigenständige Beratungsform angesehen werden (vgl. Schlüter 2010, S. 101), die die Studierenden dabei unterstützt, Potenziale und Ressourcen zu identifizieren, um individuelle (Studien-)ziele zu erreichen (vgl. Briedigkeit und Häuser 2015, S. 53). Diese Möglichkeit lässt sich sowohl auf traditionelle als auch auf „nicht-traditionelle Studienanfänger/innen“ übertragen. Unter die Kategorie ,nicht-traditionelle Studierende“ fallen unter anderem Personen, die keine allgemeine Hochschulreife im ersten Bildungsweg erlangt haben. Die meisten Universitäten und Fachhochschulen in NRW sprechen diese Zielgruppe mit der 
Möglichkeit „Studium für beruflich Qualifizierte“ an. Nach einer dreijährigen Berufsausbildung und einer mindestens zweijährigen Berufstätigkeit können auch Personen ohne eine allgemeine Hochschulreife in ihrem Studienfach studieren. Beispielsweise wird auf der Internetseite der Universität Duisburg-Essen (UDE) der Zuwachs dieser Studierendengruppe hervorgehoben, indem die UDE die Vorgabe des Ministeriums NRW mit 4\% der Vergabe der Studienplätze eines Jahrgangs anstrebt. Dies entspricht 380 Studienplätzen pro Semester. Die Zahlen vom Sommersemester 2013 zeigen einen Zuwachs gegenüber den vorherigen Jahren, allerdings liegt mit 202 Studienanfängerinnen und -anfängern ohne Abitur noch ein Entwicklungspotenzial in den Zahlen. Des Weiteren ist anzunehmen, dass sich die Lebenssituationen dieser Zielgruppe anders gestalten als die von traditionellen Studienanfängerinnen und -anfängern. Es stellt sich die Frage, wie diese Zielgruppe gezielt unterstützt werden kann und welche Hemmungen aufseiten der potenziellen Studierenden vorhanden sind. Demnach ist die Forschungsfrage, die diesem Bericht zugrunde liegt: Inwiefern kann das Mentoring-Programm Studienanfängerinnen und -anfänger dabei unterstützen, autonom ihr persönliches Studienziel zu erreichen sowie die Studieneinstiegsphase zu erleichtern? Das Instrument Mentoring kann an dieser Stelle ansetzen, um Hemmschwellen durch einen gezielten Austausch mit Studierenden zu überwinden. Nach ersten Erfahrungen in dem Mentoring-Programm der Erziehungswissenschaft und Sozialen Arbeit, die nachstehend skizziert werden, lassen sich konzeptionelle Empfehlungen auf die gestellten Fragen geben.

\section{Zur Relevanz von Mentoring-Programmen an Hochschulen}

In vielen Unternehmen und Schulen wird das Instrument Mentoring bereits eingesetzt. Daneben steigt das wissenschaftliche Interesse an der Thematik seit dem Jahr 2000. Schell-Kiehl (2005) zeigt in einer Recherchearbeit in dem „Web of Science“, (das „Web of Science“ bietet Forschenden sowie Studierenden einen Online-Zugang zu Zitationsdatenbanken), dass die Anzahl der Veröffentlichungen zu diesem Thema exponenziell angestiegen ist, wobei einige Fachdisziplinen sich erst seit Kurzem (seit ca. 2000) mit der Thematik befassen (vgl. Schell-Kiehl 2005, S. 126). In Anlehnung an diese Recherchearbeit wurde im Juli 2013 erneut eine Recherche im „Web of Science“ durchgeführt. Der Begriff Mentoring ergab eine Trefferquote von 10.925 Literaturangaben. Hierunter fallen Publikationen von 1900 bis 2013, die in dem Titel, in der Zusammenfassung oder in dem Stichwortverzeichnis den Begriff Mentoring aufweisen. Im Vergleich hatte Schell-Kiehl (2007) 1.662 Artikel angezeigt bekommen. Wird dieses Ergebnis auf den eben erwähnten Zeitraum (seit 2000 bis Juli 2013) eingegrenzt, zeigen sich folgende Zahlen. Abbildung 1 belegt, dass die Anzahl der Veröffentlichungen, die im ,Web of Science“ vermerkt sind, seit 2000 um mehr als das Doppelte gestiegen sind von 366 Veröffentlichungen auf 936 im Jahr 2010. Damit wird das Ergebnis von Schell-Kiehl (2007) bekräftigt, dass eine Steigerung hinsichtlich der Anzahl der Veröffentlichungen weiter anhält. Ebenso zeigt sich, dass die Anzahl auf diesem höheren Level seit 2010 weiterhin fortbesteht.

Wird die Anzahl hinsichtlich der einzelnen Disziplinen differenziert, zeigt sich, dass die meisten Veröffentlichungen im Bereich „Erziehungswissenschaft“ erschie- 
Abb. 1 Anzahl der Veröffentlichungen pro Jahr zu dem Suchbegriff ,Mentoring“ seit 2000 (eigene Darstellung basierend auf Daten des, Web of Science" in Anlehnung an die Studie von Schell-Kiehl 2007)

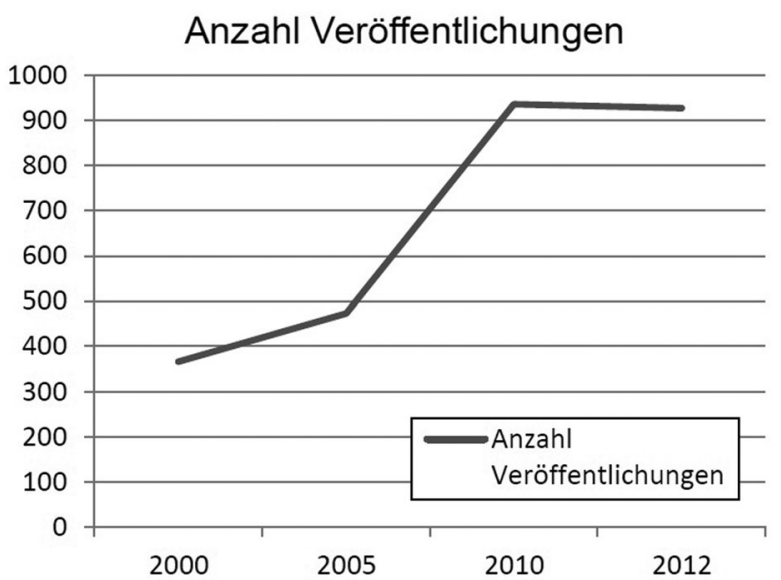

nen sind, gefolgt von den Bereichen „Management“ und „Psychologie“. Es wird deutlich, dass das wissenschaftliche Interesse an dem Instrument nicht nur entwickelt und professionalisiert wird, sondern auch eine Relevanz für die Hochschuldidaktik bietet. Wird der Suchbegriff im „Web of Science“ im Hinblick auf den Hochschulkontext eingeschränkt, ergibt sich ein Ergebnis von 805 Einträgen.

Für das im Folgenden beschriebene Mentoring-System ist zudem die regionale Entwicklung zu betrachten. Wesentlich ist hierbei die Einführung von MentoringProgrammen in NRW. Auf der Internetseite des Netzwerks Frauen- und Geschlechterforschung NRW finden sich 52 Mentoring-Programme an Hochschulen, mit den häufigsten Zielgruppen (weiblicher) wissenschaftlicher Nachwuchs, Studierende und Schüler bzw. Schülerinnen. Diese Anzahl unterstreicht ein hohes Interesse an Mentoring-Programmen als Instrument für den (erleichterten) Studienerfolg und den Gleichstellungsauftrag an Hochschulen in NRW.

Es wird ebenfalls deutlich, dass die Thematik ein aufsteigender Forschungsgegenstand für die Hochschuldidaktik geworden ist, der durch vermehrte Publikationen und Etablierung von formellen Programmen zunehmend an Bedeutung gewinnt.

\section{Das Mentoring-System an der Fakultät für Bildungswissenschaften der Universität Duisburg-Essen}

Die Auffassung, dass in Mentoring-Programmen ein Potenzial zur systematischen Studienbegleitung steckt, teilt auch die UDE. Zur Verbesserung der Beratungs- und Betreuungssituation implementierte die UDE zum Wintersemester 2009/2010 ein universitätsweites Mentoring-System, welches ein Baustein von Maßnahmen ist, um die Studienerfolgsquoten zu erhöhen und die Studienbedingungen zu verbessern. Das strukturierte Beratungsangebot fördert einen stärkeren Kontakt und eine Bindung der einzelnen Studierenden an die Universität (vgl. Stahr und Bosbach 2010).

Innerhalb der Fakultät für Bildungswissenschaften existieren unterschiedliche Ansätze für die systematische Betreuung der Studierenden. Diese orientieren sich an der Studierendenanzahl in den Studiengängen, aber auch an den Bedürfnissen und 
Tab. 1 Übersichtstabelle des Programms MEwiSA

\begin{tabular}{ll}
\hline Ziel & $\begin{array}{l}\text { Es soll eine individuelle Unterstützung und strukturelle Orientierung } \\
\text { durch ein strukturiertes Feedback zu studienbezogenen Arbeitsweisen } \\
\text { ermöglicht werden. Die Verbesserung des Lernmanagements soll durch } \\
\text { bedarfsspezifische Begleitangebote sowie die Rückmeldung der studen- } \\
\text { tischen Mentorin/des studentischen Mentors realisiert werden }\end{array}$ \\
& $\begin{array}{l}\text { Studierende in den Studienfächern Erziehungswissenschaften B.A. und } \\
\text { Soziale Arbeit B.A. ab dem 2. Semester }\end{array}$ \\
Mentees & $\begin{array}{l}\text { Studierende in den Studienfächern Erziehungswissenschaften, Erwach- } \\
\text { Mentorinnen und Mentoren }\end{array}$ \\
Betreuungsverhältnis & $1: 1$ \\
Dauer & 2 Semester \\
Instrument/e & Erstellung einer Lerntagebuchs \\
\hline
\end{tabular}

den künftigen Arbeitsfeldern der Studentinnen und Studenten. Für die Studienfächer Erziehungswissenschaft und Soziale Arbeit wurde ein Konzept entwickelt, welches sich an Studierende in ihrer Studieneingangs- und Verlaufsphase richtet. Die Tab. 1 fasst die Eckpunkte des Konzepts zusammen.

Das Programm Mentoring Erziehungswissenschaft und Soziale Arbeit (MEwiSA) ist ein studentisches Mentoring-Programm und startete im April 2012. Die Abkürzung setzt sich aus den Begriffen „Mentoring“, „Erziehungswissenschaft“ und „Soziale Arbeit“" zusammen. Die Studierenden bekommen die Möglichkeit, während ihres ersten Studienjahres von einer Mentorin oder einem Mentor begleitet zu werden. Diese/r feste Ansprechpartner/in studiert in einem höheren Fachsemester (mindestens zwei Fachsemester voraus) das gleiche Studienfach. Diese studentischen Mentorinnen und Mentoren regen die Weiterentwicklung der persönlichen und fachlichen Kompetenzen der Mentees an und dienen als Hilfestellung, damit die inhaltliche und strukturelle Orientierung der Studienanfänger im Studienverlauf erleichtert wird (vgl. Harmeier 2012, S. 4).

Dass das Mentoring-Programm in der Studieneingangsphase ansetzt, spiegelt sich auch in dem Alter der Teilnehmenden wider. In den beiden abgeschlossenen Durchgängen 2012 und 2013 lag das Durchschnittsalter bei den studentischen Mentorinnen und Mentoren bei 22,94 Jahren in einer Spanne zwischen 20 bis 38 Jahren. Bei den Mentees waren die bisherigen Teilnehmenden zwischen 18 und 39 Jahren alt. Das Durchschnittsalter entsprach traditionellen Studienanfängern mit 20,81 Jahren. Zu erwähnen ist, dass vier (17\%) von den bisherigen 24 Mentees eine Berufsausbildung abgeschlossen haben und danach ihr Studium aufnahmen. Bei den Mentorinnen und Mentoren ist ein erster Berufsabschluss vermehrt angegeben worden. Das lässt sich allerdings dadurch erklären, dass auch Masterstudierende unter den studentischen Mentoren waren, welchen ihren Bachelorabschluss als ersten Berufsabschluss definierten.

Ein weiteres Element neben der Lernprozessbegleitung ist das bedarfsspezifische Begleitprogramm. Das Begleitprogramm orientiert sich an den Wünschen der Teilnehmenden sowie an Schlüsselkompetenzen, die für die Teilnehmenden in ihrem späteren Berufsfeld verwertbar sind. Dabei werden unter anderem vorhandene Beratungs- und Serviceeinrichtungen der UDE genutzt, um ein kooperatives und strukturiertes Angebot zu gestalten (vgl. Niemann 2014, S. 38). In diesem Zusammenhang 
ist der erste Workshop vor Beginn der Lernpatenschaft für die studentischen Mentoren eine verpflichtende Qualifizierung. Diese wird von der Koordinationsstelle der Bildungswissenschaften und dem Zentrum für Hochschul- und Qualitätsentwicklung (ZfH) der UDE konzipiert und durchgeführt. Neben der Reflexion der eigenen Rolle als Mentorin oder als Mentor ist ein Lernziel der Qualifizierung, dass die studentischen Mentoren in der Lage sind, ein strukturiertes Feedback zu studienbezogenen Arbeitsweisen geben zu können (vgl. Häuser und Weihofen 2013, S. 249).

\subsection{Erste Erfahrungen der Teilnehmenden aus der Studieneingangs- und Verlaufsphase}

Inwiefern eine Unterstützung in der Studieneingangsphase von den teilnehmenden Mentees wahrgenommen wurde, zeigen erste Ergebnisse aus episodischen Interviews. Diese wurden unmittelbar nach der Teilnahme an dem Mentoring-Programm mit den Teilnehmenden durchgeführt. Relevant für die Befragung war, dass das episodische Interview einerseits auf klare Antworten abzielt, andererseits aber auch immer wieder Erzählaufforderungen stellt, um Schilderungen von bestimmten Situationen zu ermöglichen. Das episodische Interview ist als Methode geeignet, wenn „,auf Wissen, Erfahrungen und Veränderungen aus der Sicht der Befragten abgezielt werden soll, ohne jedoch einen eindeutigen und ausschließlichen Fokus auf biographische Prozesse zu legen“ (Flick 2011, S. 278). In Anlehnung an die Forschungsmethode des episodischen Interviews empfiehlt Flick (1996) die Auswertungsmethode des ,thematischen Kodierens“ (S. 160), welche daraufhin vollzogen wurde.

Nach ersten Erfahrungen aus dem Pilot-Durchgang (2012/2013) kann festgehalten werden, dass die studentischen Mentorinnen und Mentoren durch das MentoringProgramm einen Rahmen angeboten bekommen, in dem sie Theorien aus dem Studium praktisch üben können. Beratungsformen, wie Mentoring oder Kompetenzen des Beraters bzw. der Beraterin sind Studieninhalte in den angesprochenen Studiengängen der Bildungswissenschaften der Universität Duisburg-Essen (vgl. Harmeier und Häuser 2013). Aussagen von studentischen Mentoren belegen diesen Eindruck und lassen Schlüsse zu, dass die Teilnehmenden einen Nutzen im Sinne eines ,Theorie-Praxis-Transfers“" aus dem Programm ziehen (vgl. Häuser und Weihofen 2013, S. 251).

Potenzielle Vorteile für die teilnehmenden Studienanfänger lassen sich ebenfalls aus den Schilderungen der Mentees generieren. Ein häufiges Motiv für die Teilnahme war die gezielte Suche nach Hilfestellung hinsichtlich der wissenschaftlichen Arbeitsweise und studienrelevanten Themen. Nach der Teilnahme an dem einjährigen Programm formulierten die Mentees, bei sich eine positive Entwicklung in diesem Punkt erlebt zu haben. Bestätigt wird dies durch die angesprochenen Themen, die in Verlaufsprotokollen der Teilnehmenden dokumentiert werden. Die Mentees nutzen gezielt den Austausch mit der Mentorin oder dem Mentor, um die oben genannten Unsicherheiten zu kompensieren. Es lässt sich konstatieren, dass die Mentees durch den Austausch mit der Mentorin oder dem Mentor eine Erleichterung in der Studieneingangs- und Verlaufsphase erfahren haben. Die Teilnehmenden könnten alternativ den Austausch mit Familienmitgliedern oder anderen Anlaufstellen suchen. Dennoch schilderten die Mentees, dass sie besonders den Austausch mit ihrem Mentor such- 
ten, da sie diesem eine gewisse Kenntnis über die Universität zuschreiben und ihn als objektiver empfinden. Für den 2014 abgeschlossenen zweiten Durchgang zeigt sich eine ähnlich positive Perspektive.

Die Aussagen aus den ersten zwei Durchgängen lassen erkennen, dass die teilnehmenden Mentees eine Erleichterung in ihrem Studienalltag durch eine strukturierte Eingangsphase erlebten. Ebenfalls kann festgehalten werden, dass der Austausch mit einer erfahrenen Kommilitonin oder einem erfahrenen Kommilitonen Selbstvertrauen und Ermutigung für den weiteren Studienverlauf befördern kann.

\section{Erfahrungen nutzen - Perspektiven ableiten}

Es ist zu erwarten, dass der persönliche Nutzen, den die Teilnehmenden beschrieben haben, auch auf weitere Zielgruppen übertragen werden kann. Dies gilt sowohl für Studierende aus weiteren Fachbereichen als auch für ,nicht-traditionelle Studienanfänger/innen“".

\subsection{Empfehlungen für die Unterstützung im Studieneinstieg}

Der Austausch mit den Mentoren wurde als Erleichterung im Studienalltag wahrgenommen. „Nicht-traditionelle Studienanfänger/innen“ sollten demnach in Kontakt mit ebenfalls ,nicht-traditionellen Studierenden“ gebracht werden, die in dem Studienfach studieren oder bereits einen ersten Studienabschluss (z. B. Bachelor) erworben haben. Dieser Austausch kann dazu führen, Hemmungen abzubauen, da sich beide Teilnehmenden in einer ähnlichen Lebenssituation befinden. Das erlebbare Vorbild in Form des Mentors bzw. der Mentorin vermittelt Anregungen, wie ein Studienalltag mit Kindern oder einem zu pflegenden Familienmitglied gestaltet werden kann oder wie die Zeitplanung mit einer Berufstätigkeit organisiert wird. Das schafft Selbstvertrauen, um den Studienerfolg zu ermöglichen. Den emotionalen Mehrwert bestätigten bereits die befragten Mentees. Über den Austausch kann das Gefühl vermittelt werden, dass die Mentorin bzw. der Mentor die Studienfängerin bzw. den Studienanfänger versteht und unterstützend im Studienverlauf präsent ist.

Ein Mentoring-Programm an der Fachhochschule Südwestfalen greift einen weiteren Impuls aus der Befragung auf. Die Mentorinnen und Mentoren in DuisburgEssen konstatierten, dass sie eine Entwicklung der eigenen Beratungsfähigkeiten durch die Teilnahme erfahren haben. Derzeit lässt sich dieser Effekt ebenfalls im Mentoring-Programm des wissenschaftlichen Zentrums für Frühpädagogik abbilden. Beruflich qualifizierte Mentorinnen und Mentoren bilden ein Tandem mit Studierenden aus der Studieneingangs- und Verlaufsphase. Erste positive Effekte daraus sind, dass die Mentorin bzw. der Mentor eine Wertschätzung über ihre/seine Berufserfahrung erfährt sowie eine vertiefende Einübung der Beratungsmethodik erhält. Das Programm wird über eine Qualifikationsarbeit wissenschaftlich begleitet. Die ersten Ergebnisse daraus sind für das Sommersemester 2016 zu erwarten, wenn das Programm in eine weitere Praxisphase eintritt. 


\subsection{Weiterführende Empfehlungen für die Phase vor dem Studienbeginn}

Ungeachtet dessen, dass die Begleitung und Förderung in der Studieneingangsphase zentral für diesen Bericht ist, tangieren die Erfahrungen ebenfalls die Phase vor dem eigentlichen Studienbeginn. Eine weiterführende Empfehlung lässt sich auf der Ebene der Projektkoordination von Mentoring-Programmen geben. Dabei kann als Aufgabe für die Projektkoordination formuliert werden, dass neue Strategien der Teilnehmendenakquise erschlossen werden sollten. Die Erschließung von neuen Studierendengruppen bedeutet simultan auch neue Formen der Teilnehmendengewinnung durch die proaktive Ansprache von „nicht-traditionellen Studierenden“. Zum Beispiel über Mentoring-Programme, welche bereits vor Studienbeginn ansetzen und sich über den Studienbeginn fortführen lassen. Hier ist auch eine Form von Peer-Mentoring denkbar. Bei ersten Gruppentreffen vor Beginn des Studiums haben die nicht-traditionellen Studienanfängerinnen und -anfänger die Möglichkeit, direkt Fragen zu einem Studium an einen Studierenden in der gleichen Lebenssituation zu stellen. Dadurch kann auch eine Verbindung zum ersten Ansprechpartner entstehen, welche sich positiv auf den darauffolgenden eigentlichen Studienbeginn auswirken kann. Gleichermaßen entsteht eine Bindung zu der Hochschule und dem Fachbereich. Solche ersten Veranstaltungen im universitären Kontext können die Studienanfänger nutzen, um ihr Zeitmanagement vor Aufnahme eines Studiums zu überprüfen. Sie erleben die Rahmenbedingungen (Ort, Zeit, Dauer) bei einer Teilnahme an universitären Veranstaltungen und können vorab Lösungen für mögliche Barrieren finden. Durch solche Peer-Mentoring-Angebote können auch Synergie-Effekte wie gegenseitige Hilfemaßnahmen aus der Gruppe selbst entstehen. Beispiele für Hilfemaßnahmen können Lerngruppen, gegenseitige Kinderbetreuung, Fahrgemeinschaften oder der Austausch von Lebenserfahrung sein.

Diese Maßnahmen sind auf andere Fachbereiche übertragbar, weil sie die individuellen Lebensumstände und Entwicklungen der ,nicht-traditionellen Studienanfänger/innen" berücksichtigen und durch Mentorinnen und Mentoren flexibel darauf eingehen können. Die Empfehlungen verstehen sich als Impulsgeber, um vorhandene Beratungsstrukturen miteinander zu verbinden, neue zu erschließen oder bestehende Mentoring-Programme zu erweitern.

Abschließend kann festgehalten werden, dass das Potenzial, welches in MentoringProgrammen steckt, genutzt werden sollte, um „nicht-traditionellen Studierenden“ eine systematische Unterstützungsmöglichkeit während der Studieneingangsphase, aber auch während der Studienverlaufsphase zu ermöglichen. Dadurch kann nicht nur die Steigerung der Zahlen der ,nicht-traditionellen Studienanfänger/innen“ erreicht werden, sondern darüber hinaus auch der erfolgreiche Studienverlauf erleichtert werden.

Open Access Dieser Artikel wird unter der Creative Commons Namensnennung 4.0 International Lizenz (http://creativecommons.org/licenses/by/4.0/deed.de) veröffentlicht, welche die uneingeschränkte Nutzung, Verbreitung und Wiedergabe für beliebige Zwecke erlaubt, sofern Sie den/die ursprünglichen Autor(en) und die Quelle ordnungsgemäß nennen, einen Link zur Creative Commons Lizenz beifügen und angeben, ob Änderungen vorgenommen wurden. 


\section{Literatur}

Briedigkeit, E., \& Häuser, K. (2015). Praxis und Wissenschaft im Dialog. Ein Mentoring-Programm zur Systematisierung der Praxisphasen in frühpädagogischen Studiengängen. Der pädagogische Blick, 23(1), 53-57.

Flick, U. (1996). Psychologie des technischen Alltags. Soziale Konstruktion und Repräsentation technischen Wandels. Opladen: Westdeutscher Verlag.

Flick, U. (2011). Das episodische Interview. In G. Oelerich \& H.-U. Otto (Hrsg.), Empirische Forschung und Soziale Arbeit (S. 273-279). Wiesbaden: VS Verlag für Sozialwissenschaften.

Haasen, N. (2001). Mentoring- persönliche Karriereförderung als Erfolgskonzept. München: Heyne.

Harmeier, M. (2012). Mentoring in den Bildungswissenschaften. In Jahresbericht zum UDE-MentoringSystem (S. 4-5) (unveröffentl. Bericht).

Harmeier, M., \& Häuser, K. (2013). Lernprozessbegleitung durch das Mentoring-Programm MEwiSA. Personal- und Organisationsentwicklung in Einrichtungen der Lehre und Forschung, 1, 17-22.

Häuser, K., \& Weihofen, K. (2013). Das Mentoring-System in den Bildungswissenschaften: Gut qualifiziert und vorbereitet auf den Beruf durch die Arbeit als studentische Mentor/inn/en. Der pädagogische Blick, 21(4), 248-251.

Niemann, J. (2014). Managing Mentoring Concepts of Educational Sciences at the University of Duisburg-Essen. Zeitschrift für Hochschulentwicklung, 9(1), 36-44.

Schell-Kiehl, I. (2005). Zeit für Mentoring?! Zum Umgang mit Zeit in weiblichen Karrierebiographien am Beispiel von qualitativen Interviews mit Mentorinnen. In A. Schlüter (Hrsg.), In der Zeit ... Beiträge zur Biographieforschung in der Erwachsenenbildung (S. 123-147). Bielefeld: W. Bertelsmann.

Schell-Kiehl, I. (2007). Mentoring: Lernen aus Erfahrung? (Bd. 4). Bielefeld: W. Bertelsmann.

Schlüter, A. (2010). Mentoring. In A. Schlüter (Hrsg.), Bildungsberatung. Eine Einführung für Studierende (S. 101-106). Opladen: Barbara Budrich.

Stahr, I., \& Bosbach, F. (2010). Gut beraten: Das Mentoring-System der UDE. Zeitschrift für Personalund Organisationsentwicklung in Einrichtungen der Lehre und Forschung, 1, 6-11.

\section{Internetquellen}

Universität Duisburg-Essen. (2013). www.uni-due.de/beruflich-qualifizierte/index.php. Zugegriffen: 13. Mai 2014.

Kortendiek, B. (2011). www.gleichstellung-hochschulen.nrw.de. Zugegriffen: 30. April 2014. 